\title{
Fixed points of fuzzy contractive set-valued mappings and fuzzy metric completeness
}

\author{
Shihuang Hong* and Yingzi Peng
}

\section{"Correspondence:}

hongshh@hotmail.com

Institute of Applied Mathematics

and Engineering Computations,

Hangzhou Dianzi University,

Hangzhou, 310018, People's

Republic of China

\section{Springer}

\begin{abstract}
In this paper, we introduce a new fuzzy contraction via a new concept of the fuzzy sets called fw-distances initiated in the paper, which is a generalization of a fuzzy contractive mapping initiated in the article (Fuzzy Sets Syst. 159:739-744, 2008). A fixed point theorem is established by using this type of contraction of set-valued mappings in fuzzy metric spaces which are complete in the sense of George and Veeramani. As an application of our results, we give characterizations of fuzzy metric completeness. The results are supported by examples.
\end{abstract}

Keywords: fixed point; fuzzy contractive mapping; fuzzy metric completeness; fw-distance

\section{Introduction}

In Fuzzy metric spaces we refer to as KM-spaces were initiated by Kramosil and Michálek [1]. The conditions which they formulated were modified later by George and Veeramani [2] via proposing new fuzzy metric spaces called GV-spaces in this paper, with the help of continuous $t$-norms (see [3]) in order to obtain a Hausdorff topology in fuzzy metrics paces. The paper of Grabiec [4] started the investigations concerning a fixed point theory in fuzzy metric spaces by extending the well-known Banach contraction principle to KM-spaces. Many authors followed this concept by introducing and investigating the different types of fuzzy contractive mappings. Some instances of these works are in [519]. For instance, in 2002, Gregori and Sapena [5] have introduced a kind of contractive mappings and proved fuzzy fixed point theorems in GV-spaces and KM-spaces by using a strong condition for completeness, now called the completeness in the sense of Grabiec or G-completeness, which can be considered a fuzzy version of the Banach contraction theorem. These results have become recently of interest for many authors.

However, as a complete fuzzy metric space in the usual sense, that is, $M$-complete, i.e., the Cauchy sequence in the usual George and Veeramani's sense is convergent (defined, for short, $M$-Cauchy), needs not be G-complete (see $[2,6])$. Being aware of this problem, Gregori and Sapena in [5] raised the question whether the fuzzy contractive sequences are $M$-Cauchy. Very recently, many papers have appeared concerning this subject (see, for example, [7-10]). In particular, in [10], Wardowski considered a generalization of a fuzzy contractive mapping of Gregori and Sapena in $M$-complete GV-spaces, also in [7], Mihet defined a new fuzzy contraction called fuzzy $\psi$-contraction which enlarges the class of fuzzy contractive mappings of Gregori and Sapena and considered these mappings in KMspaces. They have shown that every generalized fuzzy contractive sequence is $M$-Cauchy

@2013 Hong and Peng; licensee Springer. This is an Open Access article distributed under the terms of the Creative Commons Attribution License (http://creativecommons.org/licenses/by/2.0), which permits unrestricted use, distribution, and reproduction in any medium, provided the original work is properly cited. 
in respective fuzzy metric spaces and proved fuzzy contraction fixed point theorems under different hypotheses. For instance, Mihet assumed that the space under consideration is an $M$-complete non-Archimedean KM-space. Moreover, he posed an open question whether this fixed point theorem holds if the non-Archimedean fuzzy metric space is replaced by a fuzzy metric space. Vetro [8] introduced a notion of weak non-Archimedean fuzzy metric space and proved common fixed point results for a pair of generalized contractive-type mappings. Wang [9] gave a positive answer for the open question.

Motivated by the works mentioned above, in this paper, we will establish fixed point theorems for weakly fuzzy contractive set-valued mappings on $M$-complete GV-spaces. To this end, we first introduce a new concept called $f w$-distance here. Next, using this $f w$-distance, we introduce a fuzzy $\psi$ - $p$-contractive set-valued mapping and formulate the conditions guaranteeing the convergence of a fuzzy $\psi-p$-contractive sequence and the existence of fixed points of a fuzzy $\psi$ - $p$-contractive set-valued mapping in $M$-complete GV-spaces and KM-spaces. The established notion of contraction turns out to be a generalization of the fuzzy contractive condition of Gregori and Sapena. Moreover, the paper includes a comprehensive set of examples showing that a fuzzy $\psi$-p-contractive mapping is fuzzy $\psi$-contractive and the converse is false. So our results and demonstration are also a generalization of those of $[7,9]$. To further illustrate the applicability of the $f w$-distance, we give characterizations of fuzzy metric completeness, that is, a GV-space $X$ is $M$-complete if and only if every fuzzy $\psi$ - $p$-contractive mapping from $X$ into itself has a fixed point in $X$.

Finally, the idea of the present paper has originated from the study of an analogous problem examined by Suzuki [20] for set-valued contractive mappings and [21] for singlevalued contractive mappings on complete determinacy metric spaces.

\section{Preliminaries}

Let us recall [3] that a continuous $t$-norm is a binary operation $*:[0,1] \times[0,1] \rightarrow[0,1]$ such that $([0,1], \leq, *)$ is an ordered Abelian topological monoid with unit 1 . In this sequel, we always assume that $*$ is positive, i.e., $a * b>0$ for all $a, b \in(0,1]$.

As examples of $t$-norm satisfying the conditions above, we enumerate $a * b=a b, a * b=$ $\min \{a, b\}$ and $a * b=a b / \max \{a, b, \lambda\}$ for $0<\lambda<1$, respectively.

Definition 2.1 [2] A fuzzy metric space is an ordered triple $(X, M, *)$ such that $X$ is a (nonempty) set, $*$ is a continuous $t$-norm, and $M$ is a fuzzy set on $X \times X \times(0,+\infty)$ that satisfies the following conditions for all $x, y, z \in X$ :

(F1) $M(x, y, t)>0$ for all $t>0$,

(F2) $M(x, x, t)=1$ for all $t>0$ and $M(x, y, t)=1$ for some $t>0$ implies $x=y$,

(F3) $M(x, y, t)=M(y, x, t)$ for all $t>0$,

(F4) $M(x, y, t) * M(y, z, s) \leq M(x, z, t+s)$ for all $s, t>0$ and

(F5) $M(x, y, \cdot):(0,+\infty) \rightarrow[0,1]$ is continuous.

In the definition of Kramosil and Michalek [1], $M$ is a fuzzy set on $X \times X \times[0, \infty)$ that satisfies (F3) and (F4), while (F1), (F2), (F5) are replaced by (K1), (K2), (K5), respectively, as follows:

(K1) $M(x, y, 0)=0$; 
(K2) $M(x, y, t)=1$ for all $t>0$ if and only if $x=y$;

(K5) $M(x, y, \cdot):[0, \infty) \rightarrow[0,1]$ is left continuous.

As we have mentioned, we refer to these spaces as KM-spaces and refer to the spaces given in Definition 2.1 as GV-spaces. In addition, when $X$ is called a fuzzy metric space means, it may be a GV-space or a KM-space.

In this sense, $M$ is called a fuzzy metric on $X$. Some simple but useful facts are that

(I) $M(\cdot, \cdot, t)$ is a continuous function on $X \times X$ for $t \in(0, \infty)$ and

(II) $M(x, y, \cdot)$ is nondecreasing for all $x, y \in X$.

The first fact for the proof we refer to [22, Proposition 1]. To prove the second fact, by (F4), we notice that $M(x, y, t) \geq M(x, y, s) * M(y, y, t-s)=M(x, y, s) * 1=M(x, y, s)$ for $s, t \in[0, \infty)$ with $t>s$.

Let $(X, M, *)$ be a GV-space. For $t>0$ and $r \in(0,1)$, the open ball $B(x, t, r)$ with center $x \in X$ is defined by

$$
B(x, t, r)=\{y \in X: M(x, y, t)>1-r\} .
$$

A subset $A \subset X$ is called open if for each $x \in A$, there exist $t>0$ and $0<r<1$ such that $B(x, t, r) \subset A$. Let $\mathcal{T}$ denote the family of all open subsets of $X$. Then $\mathcal{T}$ is a topology on $X$ induced by the fuzzy metric $M$. This topology is metrizable (see [23]). Therefore, $A$ closed subset $B$ of $X$ is equivalent to $x \in B$ if and only if there exists a sequence $\left\{x_{n}\right\} \subset B$ such that $\left\{x_{n}\right\}$ topologically converges to $x$. In fact, the topological convergence of sequences can be indicated by the fuzzy metric as follows.

Definition 2.2 [2] Let $(X, M, *)$ be a fuzzy metric space.

(i) A sequence $\left\{x_{n}\right\}$ in $X$ is said to be convergent to a point $x \in X$, denoted by $\lim _{n \rightarrow \infty} x_{n}=x$, if $\lim _{n \rightarrow \infty} M\left(x_{n}, x, t\right)=1$ for any $t>0$.

(ii) A sequence $\left\{x_{n}\right\}$ in $X$ is called Cauchy sequence if for each $\varepsilon>0$ and $t>0$, there exists $n_{0} \in \mathbb{N}$ such that $M\left(x_{n}, x_{m}, t\right)>1-\varepsilon$ for any $m, n \geq n_{0}$.

(iii) A fuzzy metric space $(X, M, *)$, in which every Cauchy sequence is convergent, is said to be complete.

There exist two fuzzy versions of Cauchy sequences and completeness, i.e., besides called $M$-Cauchy sequence and $M$-completeness in the sense of Definition 2.2, G-Cauchy sequence defined by $\lim _{n \rightarrow \infty} M\left(x_{n+p}, x_{n}, t\right)=1$ for all $t, p>0$ and corresponding Gcompleteness introduced by [4]. In [6], the authors have pointed out that a G-Cauchy sequence is not an $M$-Cauchy in general. It is clear that an $M$-Cauchy sequence is $G$-Cauchy, and hence, a fuzzy metric space is $M$-complete if it is $G$-complete. From now on, by Cauchy sequence and completeness we mean an $M$-Cauchy sequence and $M$-completeness.

We now introduce a new notion as follows.

Definition 2.3 Let $(X, M, *)$ be a fuzzy metric space. A fuzzy set $\mathcal{P}$ on $X \times X \times(0, \infty)$ is said to be an $f w$-distance if the following hypotheses are satisfied:

(w1) $\mathcal{P}(x, y, t) * \mathcal{P}(y, z, s) \leq \mathcal{P}(x, z, t+s)$ for all $x, y, z \in X$ and all $s, t>0$.

(w2) For any $x \in X, t \in(0, \infty), \mathcal{P}(x, \cdot, t): X \rightarrow[0,1]$ is upper semicontinuous, and $\mathcal{P}(x, y, \cdot):(0,+\infty) \rightarrow[0,1]$ is left continuous for $x, y \in X$.

(w3) Let $x, y \in X$. For any $\varepsilon \in(0,1)$ and $t>0$, there exists $\delta \in(0,1)$ and $z \in X$ such that $\mathcal{P}(z, x, t / 2) \geq 1-\delta$ and $\mathcal{P}(z, y, t / 2) \geq 1-\delta$ imply $M(x, y, t) \geq 1-\varepsilon$. 
Note that neither of the implications $\mathcal{P}(x, y, t)=1 \Leftrightarrow x=y$ (namely (F2)) necessarily hold, and $\mathcal{P}$ is nonsymmetric, i.e., in general, $\mathcal{P}$ does not satisfy (F3).

The fuzzy metric $M$ is an $f w$-distance on $\mathrm{X}$. In fact, (F4) implies that (w1) holds. The properties I and II of $M$ combining conditions (F5) or (K5) guarantee that (w2) is valid. Finally, for any $\varepsilon \in(0,1)$ and $t>0$, from the properties of $*$, we can take a small enough $\delta>0$ such that $(1-\delta) *(1-\delta) \geq 1-\varepsilon$. Now, putting $M(z, x, t / 2) \geq 1-\delta$ and $M(z, y, t / 2) \geq$ $1-\delta$, by means of $(\mathrm{F} 4)$, we have

$$
M(x, y, t) \geq M(z, x, t / 2) * M(z, y, t / 2) \geq(1-\delta) *(1-\delta) \geq 1-\varepsilon .
$$

This implies that (w3) holds. However, some other following examples of $f w$-distances show that the converse is false.

Example 2.4 Let $f: X \rightarrow \mathbb{R}_{+}$be a one-to-one continuous function, and let $g: \mathbb{R}_{+} \rightarrow$ $[0,+\infty)$ be an increasing continuous function. Define $a * b=a b$ for all $a, b \in[0,1]$. Fixed $\alpha, \beta>0$, define $M$ and $\mathcal{P}$ by, respectively,

$$
M(x, y, t)=\left(\frac{(\min \{f(x), f(y)\})^{\alpha}+g(t)}{(\max \{f(x), f(y)\})^{\alpha}+g(t)}\right)^{\beta}, \quad \mathcal{P}(x, y, t)=\left(\frac{g(t)}{(f(y))^{\alpha}+g(t)}\right)^{\beta} .
$$

Then $M$ is a fuzzy metric, and $(X, M, *)$ is a GV-space (see [24]), $\mathcal{P}$ is an $f w$-distance but not a fuzzy metric on $X$.

Proof We observe that

$$
\begin{aligned}
\mathcal{P}(x, z, t+s) & =\left(\frac{g(t+s)}{(f(z))^{\alpha}+g(t+s)}\right)^{\beta} \\
& =\left(\frac{g(t+s)}{(f(y))^{\alpha}+g(t+s)}\right)^{\beta} \cdot\left(\frac{(f(y))^{\alpha}+g(t+s)}{(f(z))^{\alpha}+g(t+s)}\right)^{\beta} \\
& \geq\left(\frac{g(t)}{(f(y))^{\alpha}+g(t)}\right)^{\beta} \cdot\left(\frac{g(s)}{(f(z))^{\alpha}+g(s)}\right)^{\beta} .
\end{aligned}
$$

Hence, $\mathcal{P}(x, z, t+s) \geq \mathcal{P}(x, y, t) * \mathcal{P}(y, z, s)$, i.e., (w1) holds. (w2) is valid. Trivial. For any $\varepsilon \in$ $(0,1)$ and $t>0$, set $\delta \in(0, \varepsilon / 3]$ and $z \in X$ such that $(1-\delta) *(1-\delta) \geq 1-\varepsilon$ and $\mathcal{P}(z, x, t / 2) \geq$ $1-\delta$ and $\mathcal{P}(z, y, t / 2) \geq 1-\delta$, we can distinguish two cases:

$$
f(x) \leq f(y) \text { and } f(y) \leq f(x)
$$

Now, we have, respectively,

$$
M(x, y, t) \geq\left(\frac{(f(x))^{\alpha}+g(t / 2)}{(f(x))^{\alpha}+g(t / 2)}\right)^{\beta}\left(\frac{(f(x))^{\alpha}+g(t / 2)}{(f(y))^{\alpha}+g(t / 2)}\right)^{\beta}
$$

and

$$
M(x, y, t) \geq\left(\frac{(f(y))^{\alpha}+g(t / 2)}{(f(y))^{\alpha}+g(t / 2)}\right)^{\beta}\left(\frac{(f(y))^{\alpha}+g(t / 2)}{(f(x))^{\alpha}+g(t / 2)}\right)^{\beta} .
$$


It is easy to verify that in the two cases, the inequality

$$
M(x, y, t) \geq \mathcal{P}(z, x, t / 2) * \mathcal{P}(z, y, t / 2) \geq(1-\delta) *(1-\delta) \geq 1-\varepsilon
$$

holds, that is, (w3) is met. This reduces that $\mathcal{P}$ is an $f w$-distance.

However, $\mathcal{P}$ is not a fuzzy metric since it is nonsymmetric.

Example 2.5 Let $X=\mathbb{R}$ with the fuzzy metric $M(x, y, t)=e^{-|x-y| / g(t)}$ with $g$ as in Example 2.4. Fixed $0<\alpha<\beta \leq 1$. Define $\mathcal{P}$ by

$$
\mathcal{P}(x, y, t)=e^{-m(x, y) / g(t)}
$$

with $m(x, y)=\max \{\alpha(x-y), \beta(y-x)\}$ and $t>0$. Then $\mathcal{P}$ is an $f w$-distance but not a fuzzy metric on $(X, M, *)$.

Some properties for the $f w$-distance are useful in this sequel.

Proposition 2.6 Let $(X, M, *)^{\prime}$ be a fuzzy metric space, and let $\mathcal{P}$ be an fw-distance on $X$. Then for sequences $\left\{x_{n}\right\}$ and $\left\{y_{n}\right\}$ in $X$, the function sequences $\left\{a_{n}(t)\right\}$ and $\left\{b_{n}(t)\right\}$ with $a_{n}, b_{n}$ : $(0, \infty) \rightarrow[0,1)$ converging to 0 for $t>0$, and $x, y, z \in X$ we have the following:

(1) if, for $t>0, \mathcal{P}\left(x_{n}, y, t / 2\right) \geq 1-a_{n}(t / 2)$ and $\mathcal{P}\left(x_{n}, z, t / 2\right) \geq 1-b_{n}(t / 2)$ for any $n \in \mathbb{N}$, then $y=z$; in particular, if $\mathcal{P}(x, y, t)=1$ and $\mathcal{P}(x, z, t)=1$, then $y=z$;

(2) if, for $t>0, \mathcal{P}\left(x_{n}, y_{n}, t / 2\right) \geq 1-a_{n}(t / 2)$ and $\mathcal{P}\left(x_{n}, z, t / 2\right) \geq 1-b_{n}(t / 2)$ for any $n \in \mathbb{N}$, then $\left\{y_{n}\right\}$ converges to $z$;

(3) if, for $t>0, \mathcal{P}\left(x_{n}, x_{m}, t / 2\right) \geq 1-a_{n}(t / 2)$ for any $n, m \in \mathbb{N}$ with $m>n$, then $\left\{x_{n}\right\}$ is a Cauchy sequence;

(4) if, for $t>0, \mathcal{P}\left(y, x_{n}, t / 2\right) \geq 1-a_{n}(t / 2)$ for any $n \in \mathbb{N}$, then $\left\{x_{n}\right\}$ is a Cauchy sequence;

(5) if $x \in X$ and $\left\{y_{n}\right\}$ in $X$ with $\lim _{n \rightarrow \infty} y_{n}=y$ and $\mathcal{P}\left(x, y_{n}, t\right) \geq \omega$ for some $\omega=\omega(x) \in(0,1)$, then $\mathcal{P}(x, y, t) \geq \omega$.

Proof (1) For any $\varepsilon \in(0,1)$ and $t>0$, let $\delta=\varepsilon$. By our assumptions, there exists $n \in \mathbb{N}$ such that $a_{n}(t / 2)<\delta$ and $b_{n}(t / 2)<\delta$ which implies that $\mathcal{P}\left(x_{n}, y, t / 2\right) \geq 1-a_{n}>1-\delta$ and $\mathcal{P}\left(x_{n}, z, t / 2\right) \geq 1-b_{n}>1-\delta$ for large enough $n$. In view of (w3), one has $M(y, z, t) \geq 1-\varepsilon$. Now, the arbitrariness of $\varepsilon$ implies that $M(y, z, t)=1$, i.e., $y=z$.

(2) Similarly to the argument of $(1)$, for any $\varepsilon \in(0,1)$ and $t>0$, we can find $n_{0} \in \mathbb{N}$ such that $M\left(y_{n}, z, t\right) \geq 1-\varepsilon$ for each $n \geq n_{0}$, that is, $\lim _{n \rightarrow \infty} y_{n}=z$.

(3) For any $\varepsilon \in(0,1)$ and $t>0$, there exists $n_{0} \in \mathbb{N}$ such that $a_{n}(t / 2)<\varepsilon$ for $n \geq n_{0}$. Let $m_{1}, m_{2} \in \mathbb{N}$ with $m_{1}, m_{2}>n>n_{0}$. Then, by means of the assumption of (3), we have $\mathcal{P}\left(x_{n}, x_{m_{1}}, t / 2\right) \geq 1-a_{n}(t / 2)$ and $\mathcal{P}\left(x_{n}, x_{m_{2}}, t / 2\right) \geq 1-a_{n}(t / 2)$. (w3) guarantees that $M\left(x_{m_{1}}, x_{m_{2}}, t\right) \geq 1-\varepsilon$. From Definition 2.2(ii) $\left\{x_{n}\right\}$ is a Cauchy sequence.

As an analogous argument in (3), we can verify that (4) is valid.

(5) If $\lim _{n \rightarrow \infty} y_{n}=y$ and $\mathcal{P}\left(x, y_{n}, t\right) \geq \omega$ for some $\omega=\omega(x) \in(0,1)$, by (w2) $\mathcal{P}(x, y, t) \geq$ $\limsup _{n \rightarrow \infty} \mathcal{P}\left(x, y_{n}, t\right) \geq \omega$. Therefore, (5) holds.

\section{Fixed point theorems}

In the sequel, by $C B(X)$, we denote the collection consisting of all nonempty closed subsets of $X$ (obviously, every closed subset of $X$ is bounded in the sense of fuzzy metric spaces). 
Let $(X, M, *)$ be a fuzzy metric space and $T: X \rightarrow C B(X)$ be a set-valued mapping. An element $x \in X$ is called a fixed point of $T$ if $x \in T x$.

The following collection $\Psi$ of functions is given in [7], that is, $\psi \in \Psi$ implies that $\psi$ from $[0,1]$ into itself is continuous, nondecreasing and $\psi(t)>t$ for each $t \in(0,1)$.

Let $\psi \in \Psi$ and $\mathcal{P}$ be an $f w$-distance. The set-valued mapping $T$ is called a fuzzy $\psi$ - $p$ contractive mapping if the following implication takes place: for any $x_{1}, x_{2} \in X$ and $y_{1} \in$ $T x_{1}$, there exists $y_{2} \in T x_{2}$ such that $\mathcal{P}\left(x_{1}, x_{2}, t\right)>0 \Rightarrow \mathcal{P}\left(y_{1}, y_{2}, t\right) \geq \psi\left(\mathcal{P}\left(x_{1}, x_{2}, t\right)\right)$ for each $t>0$. In particular, the fuzzy $\psi-M$-contraction corresponds to the fuzzy $\psi$-contraction according to [7, Definition 3.1]. A fuzzy $\psi$-p-contractive sequence in $X$ is any sequence $\left\{x_{n}\right\}$ in $X$ such that $\mathcal{P}\left(x_{n+2}, x_{n+1}, t\right) \geq \psi\left(\mathcal{P}\left(x_{n+1}, x_{n}, t\right)\right)$ for all $n \in \mathbb{N}$ and $t>0$.

Theorem 3.1 Let $(X, M, *)$ be a complete fuzzy metric space, and let $T$ be a fuzzy $\psi$ $p$-contractive set-valued mapping from $X$ into $C B(X)$. If there exists $x \in X$ such that $\mathcal{P}(x, y, t)>0$ for some $y \in T x$ and any $t>0$, then $T$ has at least a fixed point $x_{0} \in X$. Moreover, if $\mathcal{P}\left(x_{0}, x_{0}, t\right)>0$, then $\mathcal{P}\left(x_{0}, x_{0}, t\right)=1$ for all $t>0$.

Proof From our assumption, there exists $u_{0} \in X$ such that $\mathcal{P}\left(u_{0}, u_{1}, t\right)>0$ for some $u_{1} \in$ $T u_{0}$ and any $t>0$. For fixed $u_{1}$, by the contractive condition, there exists $u_{2} \in T u_{1}$ such that

$$
\mathcal{P}\left(u_{1}, u_{2}, t\right) \geq \psi\left(\mathcal{P}\left(u_{0}, u_{1}, t\right)\right) \geq \mathcal{P}\left(u_{0}, u_{1}, t\right)>0
$$

for all $t>0$. Applying again the contractive condition for $u_{1}, u_{2}$, we can choose $u_{3} \in T u_{2}$ such that

$$
\mathcal{P}\left(u_{2}, u_{3}, t\right) \geq \psi\left(\mathcal{P}\left(u_{1}, u_{2}, t\right)\right) \geq \mathcal{P}\left(u_{1}, u_{2}, t\right)>0
$$

Thus, by induction, we obtain a sequence $\left\{u_{n}\right\}$ in $X$ such that $u_{n+1} \in T u_{n}$ and

$$
\mathcal{P}\left(u_{n}, u_{n+1}, t\right) \geq \psi\left(\mathcal{P}\left(u_{n-1}, u_{n}, t\right)\right) \geq \mathcal{P}\left(u_{n-1}, u_{n}, t\right)>0
$$

for every $t>0$ and $n \in \mathbb{N}$. Next, for each $n \in \mathbb{N}$, we prove by induction that, for all $k \in \mathbb{N}$,

$$
\mathcal{P}\left(u_{n}, u_{n+k}, t\right)>0, \quad \forall t>0 .
$$

We have shown that the claim is true for $k=1$. Assume that $\mathcal{P}\left(u_{n}, u_{n+l}, t\right)>0$ for all $t>0$ and $l \in \mathbb{N}$ with $1 \leq l<k$. Then, by virtue of (w1), we have

$$
\mathcal{P}\left(u_{n}, u_{n+k}, t\right) \geq \mathcal{P}\left(u_{n}, u_{n+k-1}, t / 2\right) * \mathcal{P}\left(u_{n+k-1}, u_{n+k}, t / 2\right) .
$$

Since $\mathcal{P}\left(u_{n}, u_{n+k-1}, t / 2\right)>0, \mathcal{P}\left(u_{n+k-1}, u_{n+k}, t / 2\right)>0$, from the fact that $*$ is positive we have

$$
\mathcal{P}\left(u_{n}, u_{n+k}, t\right) \geq \mathcal{P}\left(u_{n}, u_{n+k-1}, t / 2\right) * \mathcal{P}\left(u_{n+k-1}, u_{n+k}, t / 2\right)>0
$$

for all $t>0,(1)$ is valid. 
Now, any fix $t>0$. Let $a_{n}=a_{n}(t / 2)=1-\inf _{m \geq n} \mathcal{P}\left(u_{n}, u_{m}, t / 2\right)$ for $m, n \in \mathbb{N}$. Then $\left\{a_{n}\right\}$ is a function from $(0, \infty)$ into $[0,1)$ and

$$
\mathcal{P}\left(u_{n}, u_{m}, t / 2\right) \geq 1-a_{n}
$$

for any $n, m \in \mathbb{N}$ with $m>n$. We will prove that $\left\{a_{n}\right\}$ converges to 0 . To this end, it is sufficient to verify that

$$
\lim _{n \rightarrow \infty} c_{n}=1
$$

with $c_{n}=\inf _{m \geq n} \mathcal{P}\left(u_{n}, u_{m}, t / 2\right)$. For $m \geq n+1$, by (1) we have

$$
\mathcal{P}\left(u_{n+1}, u_{m}, t / 2\right) \geq \psi\left(\mathcal{P}\left(u_{n}, u_{m-1}, t / 2\right)\right) \geq \mathcal{P}\left(u_{n}, u_{m-1}, t / 2\right) .
$$

This yields that $c_{n+1} \geq c_{n}$ for all $n \in \mathbb{N}$, i.e., $\left\{c_{n}\right\}$ is a decreasing sequence. So $\left\{c_{n}\right\}$ is convergent. Let $\lim _{n \rightarrow \infty} c_{n}=p$. By virtue of the continuity of $\psi$, we have $p \geq \psi(p)$ which yields that $p=1$, and hence (2) is valid. Moreover, by virtue of Proposition 2.6(3), we see that $\left\{u_{n}\right\}$ is a Cauchy sequence. Hence $\left\{u_{n}\right\}$ converges to a point $v_{0} \in X$ by the completeness of $X$.

Fix a large enough $n \in \mathbb{N}$. Since $\left\{u_{m}\right\}$ converges to $v_{0}$ and $\mathcal{P}\left(u_{n}, \cdot, t\right)$ is upper semicontinuous, we have

$$
\mathcal{P}\left(u_{n}, v_{0}, t\right) \geq \limsup _{m \rightarrow \infty} \mathcal{P}\left(u_{n}, u_{m}, t\right) \geq c_{n}>0
$$

This implies that

$$
\lim _{n \rightarrow \infty} \mathcal{P}\left(u_{n}, v_{0}, t\right)=1
$$

Again, the contractive hypothesis reduces that there exists $w_{n} \in T v_{0}$ such that $\mathcal{P}\left(u_{n}\right.$, $\left.w_{n}, t\right) \geq \psi\left(\mathcal{P}\left(u_{n-1}, v_{0}, t\right)\right) \geq \mathcal{P}\left(u_{n-1}, v_{0}, t\right)>0$. Consequently, we have a sequence $\left\{w_{n}\right\} \subset T v_{0}$ such that $\mathcal{P}\left(u_{n}, w_{n}, t\right)>0, \mathcal{P}\left(u_{n}, v_{0}, t\right)>0$ for all $t>0$ and $n \in \mathbb{N}$. Fix $t>0$, let

$$
a_{n}=1-\mathcal{P}\left(u_{n}, w_{n}, t / 2\right), \quad b_{n}=1-\mathcal{P}\left(u_{n}, v_{0}, t / 2\right) .
$$

In view of (4), we obtain that $\left\{b_{n}\right\}$ converges to 0 . By this, combining $\mathcal{P}\left(u_{n}, w_{n}, t / 2\right) \geq$ $\mathcal{P}\left(u_{n-1}, v_{0}, t / 2\right)$, we have $\left\{\mathcal{P}\left(u_{n}, w_{n}, t / 2\right)\right\}$ converging to 1 , which implies that $\left\{a_{n}\right\}$ converges to 0 . By Proposition 2.6(2), $\left\{w_{n}\right\}$ converges to $v_{0}$. Since $T v_{0}$ is closed, $v_{0} \in T v_{0}$, i.e., $v_{0}$ is a fixed point of $T$.

Finally, for such $v_{0}$, if $\mathcal{P}\left(v_{0}, v_{0}, t\right)>0$, there exists $v_{1} \in T v_{0}$ such that $\mathcal{P}\left(v_{0}, v_{1}, t\right) \geq$ $\psi\left(\mathcal{P}\left(v_{0}, v_{0}, t\right)\right) \geq \mathcal{P}\left(v_{0}, v_{0}, t\right)>0$. Thus, we also have a sequence $\left\{v_{n}\right\}$ in $X$ such that $v_{n+1} \in T v_{n}$ and $\mathcal{P}\left(v_{0}, v_{n+1}, t\right) \geq \psi\left(\mathcal{P}\left(v_{0}, v_{n}, t\right)\right) \geq \mathcal{P}\left(v_{0}, v_{n}, t\right)>0$ for every $n \in \mathbb{N}$. Let $a_{n}=$ $1-\mathcal{P}\left(v_{0}, v_{n}, t / 2\right)$ for fixed $t>0$. Repeating the proof process of (2), we can infer that $a_{n} \rightarrow 0$ as $n \rightarrow \infty$. By Proposition 2.6(4), $\left\{v_{n}\right\}$ is a Cauchy sequence. Hence $\left\{v_{n}\right\}$ converges to a point $x_{0} \in X$. Since $\mathcal{P}\left(v_{0}, \cdot, t\right)$ is upper semicontinuous, $1 \geq \mathcal{P}\left(v_{0}, x_{0}, t\right) \geq$ 
$\limsup _{n \rightarrow \infty} \mathcal{P}\left(v_{0}, v_{n}, t\right)=1$, and hence $\mathcal{P}\left(v_{0}, x_{0}, t\right)=1$ for all $t>0$. For any $n \in \mathbb{N}$,

$$
\mathcal{P}\left(u_{n}, x_{0}, t\right) \geq \mathcal{P}\left(u_{n}, v_{0}, t / 2\right) * \mathcal{P}\left(v_{0}, x_{0}, t / 2\right) \geq \mathcal{P}\left(u_{n}, v_{0}, t / 2\right) * 1=\mathcal{P}\left(u_{n}, v_{0}, t / 2\right) .
$$

By (4) and Proposition 2.6(1), we have $x_{0}=v_{0}$ which implies that $\mathcal{P}\left(v_{0}, v_{0}, t\right)=1$. This proof is complete.

Example 3.2 Let $X=[0, \infty), a * b=a b$ for any $a, b \in[0,1]$ and $M(x, y, t)$ be given as in Example 2.4 with $\alpha=\beta=1, g(t)=t$ and $f(x)=x$. For given $\lambda>0$, the set-valued mapping $T: X \rightarrow X$ as follows

$$
T x= \begin{cases}\{\sqrt{x+\lambda}\}, & x+\lambda \leq 1, \\ {[\sqrt{x}, \sqrt{x+\lambda}],} & x+\lambda>1\end{cases}
$$

has a fixed point in $X$.

Proof Using similar arguments as the ones in [12, Theorem 16], one can show that $(X, M, *)$ is a complete GV-space. Let $\psi(t)=\sqrt{t}$ for $t \in[0,1]$. Then $\psi \in \Psi$. Let

$$
\mathcal{P}(x, y, t)=\left\{\begin{array}{ll}
y, & 0 \leq y \leq 1, \\
\frac{1}{y}, & y>1,
\end{array} \quad x, y \in X, t>0 .\right.
$$

It is not hard to verify that $\mathcal{P}$ is an $f w$-distance. For any $x_{1}, x_{2} \in X$ and $y_{1} \in T x_{1}$, if $x_{2}+\lambda \leq 1$, then $x_{2}<1$ and choose $y_{2}=\sqrt{x_{2}+\lambda} \in T x_{2}$, we have

$$
\mathcal{P}\left(y_{1}, y_{2}, t\right)=\sqrt{x_{2}+\lambda}>\sqrt{x_{2}}=\psi\left(\mathcal{P}\left(x_{1}, x_{2}, t\right)\right) .
$$

If $x_{2}+\lambda>1$, choose $y_{2}=\sqrt{x_{2}} \in T x_{2}$, we have

$$
\mathcal{P}\left(y_{1}, y_{2}, t\right)=\sqrt{x_{2}}=\psi\left(\mathcal{P}\left(x_{1}, x_{2}, t\right)\right)
$$

if $x_{2} \leq 1$ and

$$
\mathcal{P}\left(y_{1}, y_{2}, t\right)=\frac{1}{\sqrt{x_{2}}}=\psi\left(\mathcal{P}\left(x_{1}, x_{2}, t\right)\right)
$$

if $x_{2}>1$. Consequently, $T$ is $\psi$ - $p$-contractive and all conditions of Theorem 3.1 are satisfied. Hence, $T$ has a fixed point (in fact, $1 \in T 1$ ).

Remark 3.3 We observe that $T$ in Example 3.2 is not fuzzy $\psi$-contractive. Hence, there exists a mapping which is fuzzy $\psi$ - $p$-contractive but not fuzzy $\psi$-contractive. However, every fuzzy $\psi$-contractive mapping is obviously fuzzy $\psi$ - $p$-contractive.

In fact, set $\lambda=0.005$ and take $x_{1}=0.005, x_{2}=0.04$ in Example 3.2, we have $T x_{1}=$ $\sqrt{x_{1}+\lambda}=0.1, T x_{2}=\sqrt{x_{2}+\lambda}=\sqrt{0.045}$. Note that

$$
M(0.1,0.2, t)=\frac{0.1+t}{0.2+t}, \quad M\left(x_{1}, x_{2}, t\right)=M(0.005,0.04, t)=\frac{0.005+t}{0.04+t} .
$$


Let $y_{1}=T x_{1}=0.1$ and $y_{2}=T x_{2}=\sqrt{0.045}$. Then $M\left(y_{1}, y_{2}, t\right)<M(0.1,0.2, t)$. Take $t=1$, we have

$$
M(0.1,0.2,1)=\frac{1.1}{1.2}<\sqrt{\frac{1.005}{1.04}}=\psi(M(0.005,0.04,1))=\psi\left(M\left(x_{1}, x_{2}, t\right)\right)
$$

that is, $M\left(y_{1}, y_{2}, 1\right)<\psi\left(M\left(x_{1}, x_{2}, 1\right)\right)$. Consequently, $T$ is not fuzzy $\psi$ - $M$-contractive.

Let $(X, M, *)$ be a fuzzy metric space and $T$ a single-valued mapping from $X$ into itself. $T$ is said to satisfy nonzero property if there exists $x \in X$ such that $\mathcal{P}(x, T x, t)>0$ for all $t>0$.

Corollary 3.4 Let $(X, M, *)$ be a complete fuzzy metric space, and the mapping $T$ from $X$ into itself is fuzzy $\psi$-p-contractive with the fw-distance $\mathcal{P}$ satisfying $\mathcal{P}(x, y, t)>0$ for any $(x, y, t) \in X \times X \times(0, \infty)$.If $T$ satisfies the nonzero property, then $T$ has a unique fixed point $x_{0} \in X$. Further, $x_{0}$ satisfies $\mathcal{P}\left(x_{0}, x_{0}, t\right)=1$ for all $t>0$.

Proof From Theorem 3.1, there exists $x_{0} \in X$ with $T x_{0}=x_{0}$ and $\mathcal{P}\left(x_{0}, x_{0}, t\right)=1$ for all $t>0$. Let $y_{0}=T y_{0}$. If $p\left(x_{0}, y_{0}, t\right)<1$ then $\mathcal{P}\left(x_{0}, y_{0}, t\right)=\mathcal{P}\left(T x_{0}, T y_{0}, t\right) \geq \psi\left(\mathcal{P}\left(x_{0}, y_{0}, t\right)\right)>$ $\mathcal{P}\left(x_{0}, y_{0}, t\right)>0$. This contradiction implies that $p\left(x_{0}, y_{0}, t\right)=1$. So, by $\mathcal{P}\left(x_{0}, x_{0}, t\right)=1$ and Proposition 2.6(1), we have $x_{0}=y_{0}$.

Remark 3.5 In the case of $\mathcal{P}=M, T$ is exactly fuzzy $\psi$-contractive initiated by Mihet [7]. So Corollary 3.4 is a positive answer for the open question of [7], but also an essential extension and improvement of Theorem 3.1 in [7] (see [25]) and Theorem 3 in [9], also, the corresponding results of $[5,26]$.

\section{Characterizations of completeness}

As an application of Corollary 3.4 and the $f w$-distance, we propose the following profounder result of fuzzy fixed point theory which gives characterizations of the fuzzy metric completeness. We need the following assumption:

$$
a * b \geq a b \text { for all } a, b \in(0,1]
$$

We first list the following lemmas regarding the $f w$-distance which plays a key role in this section.

Lemma 4.1 Let $X$ be a $G V$-space with the fuzzy metric $M$, let $\mathcal{P}$ be an $f w$-distance on $X$, and let $\mathcal{Q}$ be a function from $X \times X \times(0,+\infty)$ into [0,1] satisfying (w1), (w2) in Definition 2.3. Suppose that $\mathcal{Q}(x, y, t) \leq \mathcal{P}(x, y, t)$ for every $x, y \in X, t \in(0,+\infty)$. Then $\mathcal{Q}$ is also an fw-distance on $X$. In particular, if $\mathcal{Q}$ satisfies (w1), (w2) in Definition 2.3 and $\mathcal{Q}(x, y, t) \leq M(x, y, t)$ for every $x, y \in X, t \in(0,+\infty)$, then $\mathcal{Q}$ is an $f w$-distance on $X$.

Proof We show that $\mathcal{Q}$ satisfies (w3). Let $\varepsilon \in(0,1)$ and $t>0$. Since $\mathcal{P}$ is an $f w$-distance, there exists a positive number $\delta \in(0,1)$ and $z \in X$ such that $\mathcal{P}(z, x, t / 2) \geq 1-\delta$ and $\mathcal{P}(z, y, t / 2) \geq 1-\delta$ imply that $M(x, y, t) \geq 1-\varepsilon$. Then $\mathcal{Q}(z, x, t / 2) \geq 1-\delta$ and $\mathcal{Q}(z, y, t / 2) \geq$ $1-\delta$ imply that $M(x, y, t) \geq 1-\varepsilon$. This proof is complete. 
Lemma 4.2 Let $(X, M, *)$ be a $G V$-space and $A \subset X$ contain at least two points, and $c(t)=$ $\inf \{M(x, y, t): x, y \in A\}>0$. Then the fuzzy subset $p: X \times X \times(0,+\infty) \rightarrow[0,1]$ defined by

$$
p(x, y, t)= \begin{cases}M(x, y, t), & x, y \in A, \\ d(t), & \text { others }\end{cases}
$$

is an $f w$-distance on $(X, M, *)$, where $d(t)$ is a nondecreasing continuous function with $0<$ $d(t)<c(t)$.

Proof It is clear that $c(t)<1$ for $t>0$ since $A$ contains at least two points. If $x, y, z \in A$, we have

$$
\mathcal{P}(x, z, t+s)=M(x, z, t+s) \geq M(x, y, t) * M(y, z, s)=\mathcal{P}(x, y, t) * \mathcal{P}(y, z, s) .
$$

In the other case, without loss of generality, we suppose that $x \notin A$, then $\mathcal{P}(x, y, t)=d(t)$ and $\mathcal{P}(y, z, s) \leq 1$. Hence,

$$
\mathcal{P}(x, z, t+s)=d(t+s) \geq d(t) * 1 \geq p(x, y, t) * p(y, z, s) .
$$

Let $x \in X$. If $a \leq d(t)$ for some $t>0$, we have $\{y \in X: \mathcal{P}(x, y, t) \geq a\}=X$. Let $a>d(t)$ for all $t>0$. If $x \in A$, then $\mathcal{P}(x, y, t) \geq a$ implies that $y \in A$. So, we have $\{y \in X: \mathcal{P}(x, y, t) \geq a\}=$ $\{y \in X: M(x, y, t) \geq a\} \cap A$. If $x \notin A$, we have $\{y \in X: \mathcal{P}(x, y, t) \geq a\}=\emptyset$. In each case, the set $\{y \in X: \mathcal{P}(x, y, t) \geq a\}$ is closed. Therefore, $\mathcal{P}(x, \cdot, t): X \rightarrow(0,1]$ is upper semicontinuous. $\mathcal{P}(x, y, \cdot)$ is obviously continuous.

Let $\varepsilon>0$ and $t>0$. Then there exists a positive number $\tau \in(0,1 / 3)$ such that $0<\tau \varepsilon<1-$ $\sup _{t>0} d(t)$. Let $\delta=\tau \varepsilon$. Then $\mathcal{P}(z, x, t / 2) \geq 1-\delta$ and $\mathcal{P}(z, y, t / 2) \geq 1-\delta$ imply that $x, y, z \in A$. So, we have

$$
M(x, y, t) \geq M(z, x, t / 2) * M(z, y, t / 2)=\mathcal{P}(z, x, t / 2) * \mathcal{P}(z, y, t / 2) \geq(1-\delta)^{2}>1-\varepsilon .
$$

This proof is complete.

Remark 4.3 If $A$ is a compact subset of $X$, then $c(t)>0$ for all $t>0$. Indeed, suppose that this is not true, then there exists $t_{0}>0$ such that $c\left(t_{0}\right)=0$. Thus, for each $n \in \mathbb{N}$, there exist $x_{n}, y_{n} \in A$ such that $M\left(x_{n}, y_{n}, t_{0}\right)<\frac{1}{n}$. Since $A$ is compact, there exist the subsequences $\left\{x_{n_{k}}\right\},\left\{y_{n_{k}}\right\}$ of $\left\{x_{n}\right\},\left\{y_{n}\right\}$, respectively, such that $\lim _{k \rightarrow \infty} x_{n_{k}}=x$ and $\lim _{k \rightarrow \infty} y_{n_{k}}=y$ with $x, y \in A$. Note that $M$ is continuous, we have $M\left(x, y, t_{0}\right)=\lim _{k \rightarrow \infty} M\left(x_{n_{k}}, y_{n_{k}}, t_{0}\right)=0$, a contradiction. In addition, we observe that $M(x, y, \cdot)$ is nondecreasing for any given $x, y \in X$. Therefore, $c(\cdot)$ is nondecreasing, and this guarantees the existence of $d(t)$, say, $d(t)=c(t) / 2$.

Theorem 4.4 Let $(X, M, *)$ be a fuzzy metric space. Then $X$ is complete if and only if every fuzzy $\psi$-p-contractive mapping from $X$ into itself satisfying the nonzero property has a fixed point in $X$.

Proof Since the 'only if' part is proved in Corollary 3.4, we only need to prove the 'if' part. Assume that $X$ is not complete. Then there exists a sequence $\left\{x_{n}\right\}$ in $X$ which is 
Cauchy and does not converge. So, there exists $t_{n}>0$ such that $\lim _{m \rightarrow \infty} M\left(x_{n}, x_{m}, t_{n}\right)<1$ for any $n \in \mathbb{N}$. Let $S_{x}=\left\{t>0: \lim _{m \rightarrow \infty} M\left(x, x_{m}, t\right)<1\right\}$ for any $x \in X$. Moreover, we have also $\lim _{n \rightarrow \infty} \lim _{m \rightarrow \infty} M\left(x_{n}, x_{m}, t\right)=1$ for all $t>0$. Thus, any fix $i \in \mathbb{N}$, for some $n_{i}$ and each $4 t \in S_{x_{n_{i}}}$, we can choose $x_{n_{i}+1} \in\left\{x_{n}\right\}$ such that $\lim _{m \rightarrow \infty} M\left(x_{n_{i}+1}, x_{m}, t\right)>$ $\lim _{m \rightarrow \infty}\left[M\left(x_{n_{i}}, x_{m}, 4 t\right)\right]^{1 / 8}$. We now obtain a subsequence $\left\{x_{n_{i}}\right\} \subset\left\{x_{n}\right\}$ such that

$$
\lim _{j \rightarrow \infty} M\left(x_{n_{i}+1}, x_{n_{j}}, t\right)>\lim _{j \rightarrow \infty}\left[M\left(x_{n_{i}}, x_{n_{j}}, 4 t\right)\right]^{1 / 8} \quad \text { for } 4 t \in S_{x_{n_{i}}} .
$$

Let

$$
M_{\text {sup }}(x, y, t)= \begin{cases}M(x, y, t), & t<\sup S_{x} \\ M\left(x, y, \sup S_{x}\right), & t \geq \sup S_{x}\end{cases}
$$

for $\sup S_{x}<\infty$. Then we may assume that there exists a sequence $\left\{x_{n}\right\}$ in $X$ satisfying the following conditions:

(i) $\left\{x_{n}\right\}$ is Cauchy;

(ii) $\left\{x_{n}\right\}$ does not converge;

(iii) $\lim _{n \rightarrow \infty} \hat{M}\left(x_{i+1}, x_{n}, t\right)>\lim _{n \rightarrow \infty}\left[\hat{M}\left(x_{i}, x_{n}, 4 t\right)\right]^{1 / 8} \geq \hat{M}\left(x_{i}, x_{n}, t\right)$ for any $i \in \mathbb{N}, t>0$, where

$$
\hat{M}(x, y, t)= \begin{cases}M(x, y, t), & \sup S_{x}=\infty, \\ M_{\text {sup }}(x, y, t), & \sup S_{x}<\infty .\end{cases}
$$

Put $A=\left\{x_{n}: n \in \mathbb{N}\right\}$. Then $A$ is bounded and closed. We next prove that $c(t)$ given as in Lemma 4.2 is positive. In fact, if $c\left(t_{1}\right)=0$ for some $t_{1}>0$, then for every $k \in \mathbb{N}$, there exists $n(k), m(k) \in \mathbb{N}$ such that $M\left(x_{n(k)}, x_{m(k)}, t_{1}\right)<1 / k$ which implies that $\lim _{k \rightarrow \infty} M\left(x_{n(k)}\right.$, $\left.x_{m(k)}, t_{1}\right)=0$. On the other hand, since $\left\{x_{n}\right\}$ is a Cauchy sequence, we have $\lim _{k \rightarrow \infty} M\left(x_{n(k)}\right.$, $\left.x_{m(k)}, t_{1}\right)=1$, a contradiction. Hence, $c(t)>0$ for all $t>0$.

Let us define the fuzzy set $\hat{\mathcal{P}}$ on $X \times X \times(0,+\infty)$ by

$$
\hat{\mathcal{P}}(x, y, t)= \begin{cases}\hat{M}(x, y, t), & x, y \in A, \\ d(t), & x \notin A \text { or } y \notin A,\end{cases}
$$

where $d(t)$ is an increasing continuous function with $0<d(t) \leq c^{2}(t)$. It is clear that $\hat{\mathcal{P}}$ is an $f w$-distance on $X$ by Lemmas 4.1 and 4.2. Further, $\hat{\mathcal{P}}(x, y, t)=\hat{\mathcal{P}}(y, x, t)$ for any $x, y \in X$ and $t>0$, i.e., $\hat{\mathcal{P}}$ satisfies the symmetry.

Define a mapping $T: X \rightarrow X$ as follows:

$$
T x= \begin{cases}x_{1}, & x \notin A \\ x_{i+1}, & x=x_{i}(i \in \mathbb{N}) .\end{cases}
$$

Then it is easy to see that $T$ has no fixed point in $X$. Moreover, from (F1), it follows that $\hat{\mathcal{P}}\left(x_{1}, T x_{1}, t\right)=\hat{\mathcal{P}}\left(x_{1}, x_{2}, t\right)=M\left(x_{1}, x_{2}, t\right)>0$, that is, $T$ satisfies the nonzero property. To complete the proof, it is sufficient to show that $T$ is fuzzy $\psi-\hat{p}$-contractive with 
$\psi(t)=\sqrt{t} \in \Psi$. If $x \notin A$ or $y \notin A$, then

$$
\hat{\mathcal{P}}(T x, T y, t) \geq c(t) \geq \sqrt{d(t)}=\psi(\hat{\mathcal{P}}(x, y, t)) .
$$

Let us assume that $x, y \in A$. Then, without loss of generality, we may assume that $x=x_{i}$, $y=x_{j}$ and $i<j$. For any $t>0$, from (iii), combining the monotonicity of $\hat{M}(x, y, \cdot)$, it follows that

$$
\begin{aligned}
\lim _{n \rightarrow \infty} \hat{M}\left(x_{i}, x_{n}, 2 t\right) & \geq \hat{M}\left(x_{i}, x_{j}, t\right) * \lim _{n \rightarrow \infty} \hat{M}\left(x_{j}, x_{n}, t\right) \\
& \geq \hat{M}\left(x_{i}, x_{j}, t\right) * \lim _{n \rightarrow \infty} \hat{M}\left(x_{j-1}, x_{n}, t\right) \geq \cdots \\
& \geq \hat{M}\left(x_{i}, x_{j}, t\right) * \lim _{n \rightarrow \infty} \hat{M}\left(x_{i+1}, x_{n}, t\right) \\
& \geq \hat{M}\left(x_{i}, x_{j}, t\right) * \lim _{n \rightarrow \infty}\left[\hat{M}\left(x_{i}, x_{n}, 4 t\right)\right]^{1 / 8} \\
& \geq \hat{M}\left(x_{i}, x_{j}, t\right) * \lim _{n \rightarrow \infty}\left[\hat{M}\left(x_{i}, x_{n}, 2 t\right)\right]^{1 / 8} \\
& \geq \hat{M}\left(x_{i}, x_{j}, t\right) \lim _{n \rightarrow \infty} \sqrt{\hat{M}\left(x_{i}, x_{n}, 2 t\right)} .
\end{aligned}
$$

This implies that

$$
\lim _{n \rightarrow \infty} \sqrt{\hat{M}\left(x_{i}, x_{n}, 2 t\right)} \geq \hat{M}\left(x_{i}, x_{j}, t\right) .
$$

On the other hand, by (iii), combining (4) and the symmetry of $\hat{\mathcal{P}}$, we have

$$
\begin{aligned}
\hat{\mathcal{P}}\left(T x_{i}, T x_{j}, t\right) & =\hat{\mathcal{P}}\left(x_{i+1}, x_{j+1}, t\right)=\hat{M}\left(x_{i+1}, x_{j+1}, t\right) \\
& \geq \lim _{n \rightarrow \infty} \hat{M}\left(x_{i+1}, x_{n}, t / 2\right) * \lim _{n \rightarrow \infty} \hat{M}\left(x_{j+1}, x_{n}, t / 2\right) \\
& \geq \lim _{n \rightarrow \infty} \hat{M}\left(x_{i+1}, x_{n}, t / 2\right) * \lim _{n \rightarrow \infty} \hat{M}\left(x_{j}, x_{n}, t / 2\right) \geq \cdots \\
& \geq \lim _{n \rightarrow \infty} \hat{M}\left(x_{i+1}, x_{n}, t / 2\right) * \lim _{n \rightarrow \infty} \hat{M}\left(x_{i+1}, x_{n}, t / 2\right) \\
& \geq\left[\lim _{n \rightarrow \infty} \hat{M}\left(x_{i+1}, x_{n}, t / 2\right)\right]^{2} \geq\left[\lim _{n \rightarrow \infty} \hat{M}\left(x_{i}, x_{n}, 2 t\right)\right]^{1 / 4} \\
& \geq \sqrt{\hat{M}\left(x_{i}, x_{j}, t\right)}=\psi\left(\hat{M}\left(x_{i}, x_{j}, t\right)\right)=\psi\left(\hat{\mathcal{P}}\left(x_{i}, x_{j}, t\right)\right) .
\end{aligned}
$$

This shows that $T$ is fuzzy $\psi-\hat{p}$-contractive.

Example 4.5 For any nonempty set $X$, let us consider the fuzzy metric space $(X, M, *)$ with $M(x, y, t)$ as in Example 3.2. Let $X_{a}=(a, \infty)$ for any fixed $a>1$. Then the fuzzy metric space $\left(X_{a}, M, *\right)$ is not complete.

Proof Consider the mapping $T x=\sqrt{x+\lambda}$ with $0<\lambda \leq a(a-1)$ and $\psi(t)=\sqrt{t}$, we have $\psi \in \Psi$. Let $\mathcal{P}(x, y, t)=\frac{\min \{x, y\}}{\max \{x, y\}}$, we have $\mathcal{P}(x, y, t) \leq M(x, y, t)$ for any $x, y \in X$ and $t>0$. From Lemma 4.1, it follows that $\mathcal{P}$ is an $f w$-distance in $X$. Now, we have $\mathcal{P}(x, T x, t)=\frac{\min \{x, \sqrt{x+1}\}}{\max \{x, \sqrt{x+1}\}}>$ 
0 for all $t>0$ and

$$
\mathcal{P}(T x, T y, t)=\frac{\sqrt{x+1}}{\sqrt{y+1}}>\sqrt{\frac{x}{y}}=\psi(\mathcal{P}(x, y, t))
$$

for $x \leq y$. This implies that $T$ is fuzzy $\psi-p$-contractive. We assert that $T$ has no fixed point in $X_{a}$. Indeed, if $x=T x$, then $x=\frac{1+\sqrt{1+4 \lambda}}{2} \leq a$. Consequently, Theorem 4.4 guarantees that $\left(X_{a}, M, *\right)$ is not complete.

\section{Competing interests}

The authors declare that they have no competing interests.

\section{Authors' contributions}

All authors contributed equally and significantly in writing this article. All authors read and approved the final manuscript.

\section{Acknowledgements}

Supported by the Natural Science Foundation of Zhejiang Province (LY12A01002).

Received: 24 June 2013 Accepted: 10 September 2013 Published: 08 Nov 2013

\section{References}

1. Kramosil, I, Michálek, J: Fuzzy metrics and statistical metric spaces. Kybernetika 11, 326-334 (1975)

2. George, A, Veeramani, P: On some results in fuzzy metric spaces. Fuzzy Sets Syst. 64, 395-399 (1994)

3. Hadžić, O, Pap, E: Fixed Point Theory in Probabilistic Metric Space. Kluwer Academic, Dordrecht (2001)

4. Grabiec, M: Fixed points in fuzzy metric spaces. Fuzzy Sets Syst. 27, 385-389 (1988)

5. Gregori, V, Sapena, A: On fixed-point theorems in fuzzy metric spaces. Fuzzy Sets Syst. 125, 245-252 (2002)

6. Vasuki, R, Veeramani, P: Fixed point theorems and Cauchy sequences in fuzzy metric spaces. Fuzzy Sets Syst. 135, 415-417 (2003)

7. Mihet, D: Fuzzy $\psi$-contractive mappings in non-Archimedean fuzzy metric spaces. Fuzzy Sets Syst. 159, 739-744 (2008)

8. Vetro, C: Fixed points in weak non-Archimedean fuzzy metric spaces. Fuzzy Sets Syst. 162, 84-90 (2011)

9. Wang, S: Answers to some open questions on fuzzy $\psi$-contractions in fuzzy metric spaces. Fuzzy Sets Syst. 222, 115-119 (2013)

10. Wardowski, D: Fuzzy contractive mappings and fixed points in fuzzy metric spaces. Fuzzy Sets Syst. 222, 108-114 (2013)

11. Zhu, X-H, Xiao, J-Z: Note on 'Coupled fixed point theorems for contractions in fuzzy metric spaces'. Nonlinear Anal. 74, 5475-5479 (2011)

12. Gregori, V, Minana, J, Morillas, S: Some questions in fuzzy metric spaces. Fuzzy Sets Syst. 204, 71-85 (2012)

13. Xiao, J, Zhu, X, Jin, X: Fixed point theorems for nonlinear contractions in Kaleva-Seikkala's type fuzzy metric spaces. Fuzzy Sets Syst. 200, 65-83 (2012)

14. Roldán, A, Moreno, J, Roldán, C: Tripled fixed point theorem in fuzzy metric spaces and applications. Fixed Point Theory Appl. (2013). doi:10.1186/1687-1812-2013-29

15. Ćirić, LB: Some new results for Banach contractions and Edelstein contractive mappings on fuzzy metric spaces Chaos Solitons Fractals 42, 146-154 (2009)

16. Razani, A: A contraction theorem in fuzzy metric space. Fixed Point Theory Appl. 2005, 257-265 (2005). doi:10.1155/FPTA.2005.257

17. Hadžić, O, Pap, E: A fixed point theorem for multivalued mappings in probabilistic metric spaces and an application in fuzzy metric spaces. Fuzzy Sets Syst. 127, 333-344 (2002)

18. Kiany, F, Amini-Harandi, A: Fixed point and endpoint theorems for set-valued fuzzy contraction maps in fuzzy metric spaces. Fixed Point Theory Appl. 2011, Article ID 94 (2011). doi:10.1186/1687-1812-2011-94

19. Arshad, M, Shoaib, A: Fixed points of a multivalued mappings in fuzzy metric spaces. In: Proceedings of the World Congress on Engineering, vol. I. WCE 2012, July 4-6, London, U.K. (2012)

20. Suzuki, T, Takahashi, W: Fixed point theorems and characterizations of metric completeness. Topol. Methods Nonlinear Anal. 8, 371-382 (1996)

21. Suzuki, T: A generalized Banach contraction principle that characterizes metric completeness. Proc. Am. Math. Soc. $136,1861-1869(2008)$

22. Rodríguez-Lôpez, J, Romaguera, S: The Hausdorff fuzzy metric on compact sets. Fuzzy Sets Syst. 147, 273-283 (2004)

23. Gregori, V, Romaguera, S: Some properties of fuzzy metric spaces. Fuzzy Sets Syst. 115, 485-498 (2000)

24. Gregori, V, Morillas, S, Sapena, A: Examples of fuzzy metrics and applications. Fuzzy Sets Syst. 170, 95-111 (2011)

25. Mihet, D: Erratum to 'Fuzzy $\psi$-contractive mappings in non-Archimedean fuzzy metric spaces revisited'. Fuzzy Sets Syst. 161, 1150-1151 (2010) (Fuzzy Sets Syst. 159, 739-744 (2008))

26. Radu, V: Some remarks on the probabilistic contractions on fuzzy Menger spaces. The Eighth International Conference on Appl. Math. Comput. Sci., Cluj-Napoca, 2002. Autom. Comput. Appl. Math. 11, 125-131 (2002) 